\title{
Risk factors for Anopheles mosquitoes in rural and urban areas of Blantyre District, southern Malawi
}

\section{Themba Mzilahowa ${ }^{1}$, Madalitso Luka-Banda ${ }^{1}$, Veronica Uzalili ${ }^{1}$, Don P. Mathanga ${ }^{1}$, Carl H. Campbell Jr ${ }^{2}$, Mavuto Mukaka ${ }^{3}$, John E. Gimnig ${ }^{4}$}

\author{
1. Malaria Alert Centre, College of Medicine, University of Malawi, Blantyre, Malawi \\ 2. Center for Tropical and Emerging Global Diseases, University of Georgia, Athens, Georgia, USA \\ 3. Malawi-Liverpool-Wellcome Trust Clinical Research Programme, Blantyre, Malawi \\ 4. Entomology Branch, Division of Parasitic Diseases and Malaria, Centers for Disease Control and Prevention, Atlanta, Georgia, USA
}

Correspondence: Dr Themba Mzilahowa (tmzilahowa@mac.medcol.mw)

\section{Background}

\section{Abstract}

Although urban malaria transmission is low and seasonal, it remains a major public health problem. This study aimed at demonstrating the presence of Anopheles mosquitoes and their potential to transmit malaria in urban settings.

Methods

Two cross-sectional surveys were carried out in Blantyre District, Malawi, during the dry and wet seasons of 2008 and 2010, respectively. A map of Blantyre was divided into a grid of 400 cells, of which 60 cells were randomly selected. Five households located within $100 \mathrm{~m}$ from the centre of each selected cell were enrolled, a standard questionnaire was administered, and indoor resting mosquitoes were sampled.

Results

In 2008 and 2010, a total of 960 and 1045 mosquitoes were collected, respectively. Anopheles funestus comprised $9.9 \%(\mathrm{n}=95)$ and $10.3 \%$ ( $\mathrm{n}=108)$ during the two surveys, respectively. Anopheles gambiae sensu lato (s.l.) was rarely detected during the second survey ( $\mathrm{n}=6 ; 0.6 \%$ ). Molecular identification was performed on samples collected during the first survey, and An. funestus sensu stricto (s.s.) was the only sibling species detected. All the Anopheles mosquitoes were collected from households located in rural areas of Blantyre and none from urban areas.

In univariate analysis, the presence of open eaves was associated with increased Anopheles prevalence, both during the dry (incidence rate ratio, IRR $=$ 4.3; 95\% CI 2.4 - 7.6) and wet (IRR = 2.47; 95\% CI 1.7 - 3.59) seasons. Chances of detecting Anopheles spp. decreased with increasing altitude (IRR = $0.996 ; 95 \%$ CI $0.995-0.997$ ) and during the dry season, but increased during the wet season (IRR $=1.0017$; $95 \%$ CI $1.0012-1.0023$ ). These factors remained significant following a multiple Poisson regression analysis. No association was found between insecticide-treated bednet ownership and the number of Anopheles mosquitoes detected.

\section{Conclusions}

The presence of An. funestus s.s and An. gambiae s.l. in the periphery of Blantyre city was an indication that malaria transmission was potentially taking place in these areas.

\section{Introduction}

In most urban settings across sub-Saharan Africa, malaria transmission and parasite prevalence are generally low and seasonal, with most of the transmission taking place in the wet season. ${ }^{1-5}$ Even with such low and seasonal disease transmission, malaria continues to pose a serious challenge to urban-dwelling communities, both in terms of disease severity and the number of reported cases. The sources of malaria infections observed among urban-dwelling populations likely varies from city to city. In some areas, vector breeding has been documented in urban settings with measurable entomological inoculation rates (EIRs). ${ }^{6-8}$ Other studies have suggested that urban-rural migration is a major source of the malaria cases reported or detected in urban areas. ${ }^{9-11}$ In either case, it is important for malaria control programmes to know the contribution of each potential source of malaria infection. Such knowledge is vital to designing better and more suitable control strategies.

Malaria is a common disease in Blantyre District in southern Malawi, including urban areas of Blantyre, which account for nearly $60 \%$ of the population of the district. Like the rest of sub-Saharan Africa, malaria transmission in Malawi is largely sustained by two efficient vectors: Anopheles gambiae sensu lato (s.l.) and Anopheles funestus. There are, however, notable differences in endemicity of the disease across the country, with lakeshore and low-lying areas experiencing intense and perennial malaria transmission (holoendemic), while upland areas are characterised by seasonal transmission coinciding with the wet season. ${ }^{12,13}$ Epidemiological studies have suggested that the strongest risk factor for malaria acquisition among urban residents in Blantyre is a history of travel to rural areas, ${ }^{9}$ but there is potential for local malaria transmission due to mosquito breeding in isolated pockets across the city. On the other hand, if most transmission is occurring among travellers outside the city, then vector control targeting urban areas would have limited impact. This study was carried out, therefore, to assess the presence and number of Anopheles vector species in urban areas of Blantyre, in comparison to the rural areas, as a proxy indicator for malaria transmission.

\section{Methods}

\section{Study setting}

Blantyre District has an estimated total population of 1,001,984 people, with 661,256 people residing in urban Blantyre city. The district is located in the Shire Highlands and lies at $15^{\circ} 42^{\prime} \mathrm{S}$ and $35^{\circ} \mathrm{E}$. The city is surrounded by four mountains with varying topography, ranging from about 780 $\mathrm{m}$ to $1612 \mathrm{~m}$ above sea level. ${ }^{14}$

Blantyre has a tropical continental climate, with two distinct seasons in the year. The wet season is from November to April, with continuing light cold showers from May to July. The dry season is from May to October. The mean annual rainfall is $1122 \mathrm{~mm}$, of which about $80 \%$ falls between November and March. The city is generally cool with mean annual temperatures ranging from $13{ }^{\circ} \mathrm{C}\left(55^{\circ} \mathrm{F}\right)$ during the cold season (May to July) to $21^{\circ} \mathrm{C}\left(70{ }^{\circ} \mathrm{F}\right)$ during the hot season (September to November). ${ }^{14}$ Blantyre is served by a number of health facilities, both private and governmentowned. It has one major referral hospital (Queen Elizabeth Central Hospital) and two major private hospitals. Besides these, there are additional health facilities in all the major townships within the city and rural areas in the district. 


\section{Study sites selection}

A grid with $500 \mathrm{~m} \times 500 \mathrm{~m}$ cells was drawn over a map of Blantyre city and the surrounding area to generate a sampling frame (Figure 1). The total area of the grid was approximately $100 \mathrm{~km}^{2}$, with a total of 400 grid cells. Many of the cells were in completely unpopulated areas and were therefore ineligible for sampling. Sixty cells were randomly selected and teams went to the approximate centre of each cell using geographical positioning system (GPS) navigation. In each cell, 5 houses nearest to the centre and within 100 $\mathrm{m}$ were selected for household characteristic data collection and mosquito sampling. Urban areas were defined as areas located within the city boundaries, while all households located outside these official boundaries were classified as rural.

Figure 1: Map of Blantyre district overlaid with a grid containing cells measuring $500 \mathrm{~m} \times 500 \mathrm{~m}$ as sampling points

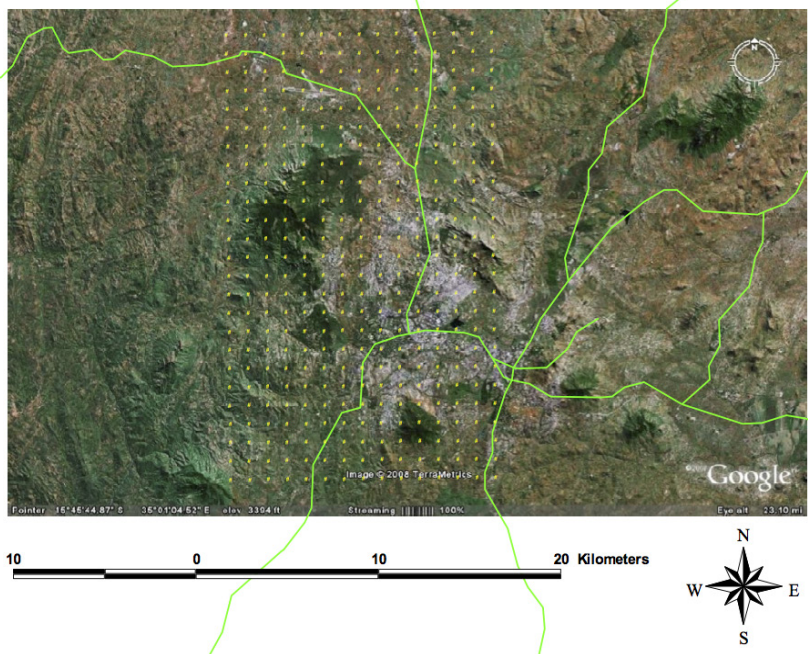

\section{Field data collection}

There were two teams of five people in each cell. Each team had two personal digital assistants (PDAs) with GPS units (Dell Axim X50s, Dell Inc., Austin, TX, USA), a backpack aspirator (John W. Hock Company; Florida, USA) powered by a $12 \mathrm{~V}$ rechargeable battery (Power SP Sonic, Model PS $12180 \mathrm{NB}$ ), a set of petri dishes, and field notebooks. Using PDAs and GPS sample software,,$^{15}$ each team navigated to a preselected cell until they reached the approximate centre. Five houses nearest to the centre in each of the randomly selected cells were sampled for the presence of Anopheles mosquitoes using a backpack aspirator.

At the time of sampling, housing characteristics, including roof type, wall type, house eaves, and windows were also recorded, along with the number of people sleeping in the house and the number of insecticide-treated bednets (ITNs) in the house.

Before mosquito collection was carried out, written informed consent was obtained from the house owners for permission to enter their houses for mosquito collection and to conduct interviews using a standard questionnaire. The first survey was carried out during the dry season of November 2008 and the second one at the end of the wet season, in April 2010. This study was approved by the Malawi College of Medicine Research and Ethics Committee (COMREC).

\section{Mosquito collection}

Motorised backpack aspirators were used to collect mosquitoes. All mosquitoes collected from individual houses were put in separate petri dishes, labelled and transported in cooler boxes to the Malaria Alert Centre (MAC) laboratory, in Blantyre, where specimens were counted and morphologically identified using standard morphological identification keys. ${ }^{16}$ A subset of Anopheles funestus s.l. specimens collected from the first (2008) survey were submitted for further molecular identification of the individual member species by polymerase chain reaction (PCR).

\section{Molecular identification of Anopheles spp.}

Molecular analyses were carried out on a sample of 48 mosquitoes morphologically identified as An. funestus s.l.. PCR was run for An. gambiae s.l., as well, to rule out any misidentifications. ${ }^{17-19}$ Briefly, a multiplex PCR was run to discriminate members of the An. funestus group in $25 \mu \mathrm{L}$ reactions. The cocktail comprised a forward universal primer and six reverse primers capable of identifying only five of the ten members of the An. funestus group of mosquitoes, namely, An. funestus sensu stricto (s.s.), An. parensis, An. rivulorum, An. leesoni, and An. vaneedeni. Primers for the other members of this species complex were not included because they are not important in disease transmission and are scarce in the local environment. All reactions were run with both positive and negative controls. The PCR cycle conditions were: an initial denaturation at $94^{\circ} \mathrm{C}$ for 4 minutes for 1 cycle, followed by 30 cycles at $94^{\circ} \mathrm{C}$ for 30 seconds, $58^{\circ} \mathrm{C}$ for 30 seconds, and $72^{\circ} \mathrm{C}$ for 45 seconds, and a final annealing cycle at $72^{\circ} \mathrm{C}$ for 7 minutes.

\section{Data analysis}

All mosquitoes collected were recorded as counts. The various species detected were summarised as proportions. A multiple Poisson regression model was carried out to assess if the various factors (open eaves, altitude, window type, and presence of ITNs) included in the data set were independently associated with Anopheles abundance.

\section{Results}

\section{Household characteristics}

Household characteristics from the first (2008) and second (2010) surveys are summarised in Table 1. A total of 250 and 206 households were sampled during the respective surveys. In both surveys, more rural households were sampled $(70.8 \%$; $68.9 \%)$ and fewer $(29.2 \% ; 31.1 \%)$ from urban areas. Slightly more than half $(53.6 \% ; 52.8 \%)$ of the sampled rural houses had a thatched roof during the first and second surveys, respectively. Although more houses in urban areas had iron roofs $(97.6 \% ; 89.1 \%)$, a few $(2.9 \% ; 10.9 \%)$ had thatched roofs. Overall, more than half of the houses sampled had sealed eaves $(\mathrm{n}=122,55.2 \% ; \mathrm{n}=130,63.1 \%)$ during the two surveys. In both surveys (2008; 2010), a significantly higher proportion of houses ( $\mathrm{n}=63,92.7 \% ; \mathrm{n}=52,81.3 \%$ )

Table 1: Household characteristics from 2008 and 2010 surveys

\begin{tabular}{|c|c|c|c|c|c|c|}
\hline \multirow{2}{*}{ Characteristics } & \multicolumn{3}{|c|}{$\begin{array}{r}2008 \\
\text { n (\%) }\end{array}$} & \multicolumn{3}{|c|}{$\begin{array}{r}2010 \\
\text { n (\%) }\end{array}$} \\
\hline & Rural & Urban & Total & Rural & Urban & Total \\
\hline Households & $177(70.8)$ & $73(29.2)$ & $250(100)$ & $142(68.9)$ & $64(31.1)$ & $206(100)$ \\
\hline \multicolumn{7}{|l|}{ Roof type } \\
\hline Thatched & $82(53.6)$ & $2(2.9)$ & $84(38)$ & $75(52.8)$ & $7(10.9)$ & $82(39.8)$ \\
\hline Iron sheet & $71(46.4)$ & $66(97.6)$ & $137(62)$ & $67(47.2)$ & $57(89.1)$ & $124(60.2)$ \\
\hline \multicolumn{7}{|l|}{ Eaves } \\
\hline Open & $94(61.4)$ & $5(7.4)$ & $99(44.8)$ & $64(45.1)$ & $12(18.8)$ & $76(36.9)$ \\
\hline Sealed & $59(38.6)$ & $63(92.7)$ & $122(55.2)$ & $78(54.9)$ & $52(81.3)$ & $130(63.1)$ \\
\hline ITN ownership & $106(69.3)$ & $47(69.1)$ & $153(69.2)$ & $121(85.2)$ & $50(78.1)$ & $171(83.0)$ \\
\hline Net retreatment & $98(92.3)$ & $44(93.6)$ & $142(92.8)$ & 83 (68.6) & $46(92.0)$ & $129(75.4)$ \\
\hline
\end{tabular}


Table 2: Total number of mosquitoes captured in each survey and mosquito density per house

\begin{tabular}{|c|c|c|c|c|c|c|}
\hline \multirow{2}{*}{$\begin{array}{l}\text { Mosquito } \\
\text { species }\end{array}$} & \multicolumn{3}{|c|}{$\begin{array}{l}2008 \\
\text { n (\%) }\end{array}$} & \multicolumn{3}{|c|}{$\begin{array}{l}2010 \\
\text { n (\%) }\end{array}$} \\
\hline & Rural & Urban & Total & Rural & Urban & Total \\
\hline An. gambiae s.l. & $0(0.0)$ & $0(0.0)$ & $0(0.0)$ & $6(0.9)$ & $0(0.0)$ & $6(0.6)$ \\
\hline An. funestus s.l. & 95 (29.9) & $0(0.0)$ & $95(10.3)$ & $108(16.1)$ & $0(0.0)$ & $108(10.3)$ \\
\hline Culex spp. & $223(70.1)$ & $602(100)$ & $825(89.7)$ & $542(80.7)$ & $373(100)$ & 915 (87.6) \\
\hline Mansonia spp. & $0(0.0)$ & $0(0.0)$ & $0(0.0)$ & $16(2.4)$ & $0(0.0)$ & $16(1.5)$ \\
\hline Aedes spp. & $0(0.0)$ & $0(0.0)$ & $0(0.0)$ & $0(0.0)$ & $0(0.0)$ & $0(0.0)$ \\
\hline Total & 318 & 602 & 920 & 672 & 373 & 1045 \\
\hline No. of houses & 197 & 53 & 250 & 142 & 64 & 206 \\
\hline $\begin{array}{l}\text { Mosquitoes/ } \\
\text { house }\end{array}$ & 1.6 & 11.4 & 3.7 & 4.7 & 5.8 & 5.1 \\
\hline
\end{tabular}

located in urban areas had sealed eaves. More houses located in rural areas characteristically had open eaves both in 2008 $(\mathrm{n}=94,61.4 \%)$ and in $2010(\mathrm{n}=64,45.1 \%)$.

ITN ownership was generally high $(\mathrm{n}=153,69.2 \%$; $\mathrm{n}=$ $154,83.0 \%$ ) during the first and second surveys, respectively, and the majority of the nets owned had been retreated with an insecticide $(92.2 \% ; 75.4 \%)$, following the routine net retreatment campaigns implemented at the time.

\section{Mosquito species abundance}

The number of mosquitoes captured in rural and urban households in 2008 and 2010 is summarised in Table 2. The two surveys $(2008 ; 2010)$ yielded a total of 920 and 1045 mosquitoes, respectively. In both surveys, the bulk of the mosquitoes ( $\mathrm{n}=825,89.7 \% ; \mathrm{n}=915,87.6 \%$ ) found resting in people's homes were Culex spp., which were equally abundant in both rural and urban areas. Of the anophelines collected, An. funestus was the most common species ( $\mathrm{n}=95,10.3 \%$; $\mathrm{n}$ $=108,10.3 \%$ ) detected during both surveys. An. gambiae s.l was only detected in the 2010 survey ( $n=6,0.6 \%$ ). Of the mosquitoes identified morphologically as An. funestus, 14/48 (29.2\%) specimens were identified as An. funestus s.s. by PCR from the first survey collections. However, the majority of the specimens $(\mathrm{n}=34,70.8 \%)$ did not amplify. No further molecular identifications were carried out on the specimens collected during the second survey.

\section{Factors associated with Anopheles presence}

By univariate analysis, several factors (presence of ITNs, type of windows [open or closed], house eaves [open or sealed], and elevation) were analysed for association with presence of Anopheles mosquitoes by Poisson regression (Table 3). ITN ownership was not associated with the number of Anopheles mosquitoes in either survey, while having closed windows was significantly associated with fewer Anopheles mosquitoes (incidence rate ratio, $\mathrm{IRR}_{1}=0.53,95 \%$ confidence interval, $\mathrm{CI}=0.33$ to $0.85 ; \mathrm{IRR}_{2}=0.60,95 \% \mathrm{CI}=0.37$ to 0.98$)$. In both surveys (2008; 2010), houses with open eaves were more likely to harbour Anopheles spp. $\left(\mathrm{IRR}_{1}=4.3,95 \% \mathrm{CI}\right.$ $=2.4$ to $7.6 ; \mathrm{IRR}_{2}=2.47,95 \% \mathrm{CI}=1.7$ to $\left.3.59 ; \mathrm{P}<0.001\right)$ compared to houses with closed eaves. Although altitude was significantly associated with incidence of Anopheles mosquitoes in both surveys, the direction of association was different in the two surveys. Decreasing altitude was associated with increasing number of Anopheles $\left(\mathrm{IRR}_{1}=\right.$ $0.996,95 \% \mathrm{CI}=0.995$ to 0.997 ) during the first survey while in the follow-up survey, increasing altitude was associated with increased number of Anopheles mosquitoes $\left(\mathrm{IRR}_{2}=\right.$ $1.0017,95 \%=$ CI 1.0012 to 1.0023$)$.

In a multivariate Poisson regression, two factors (house eaves and altitude) remained significantly associated with http://dx.doi.org/10.4314/mmj.v28i4.2 incidence of Anopheles spp. after performing multiple Poisson regression and adjusting for other factors (Table 3). Open eaves were significantly associated with increased numbers of Anopheles mosquitoes during the two surveys $\left(\mathrm{IRR}_{1}=3.5,95 \% \mathrm{CI}=1.5\right.$ to $8.1 ; \mathrm{IRR}_{2}=2.08,95 \% \mathrm{CI}=$ 1.48 to 3.04 ), while increasing elevation was associated with fewer mosquitoes in 2008 but more mosquitoes in 2010 (RR $=0.997,95 \% \mathrm{CI}=0.996$ to $0.998 ; \mathrm{IRR}_{2}=1.002,95 \% \mathrm{CI}=$ 1.001 to 1.002$)$.

\section{Discussion}

The results of this study show that two known malaria vectors, An. funestus and An. gambiae s.l., exist in Blantyre District in southern Malawi. The former was found during both surveys (dry and wet seasons), while the latter was only detected in low numbers in the second survey (at the end of the wet season). In this study, the two Anopheles species were observed only in rural areas. These findings indicate that people living in rural or peripheral areas in Blantyre are more exposed to Anopheles mosquito bites than urban inhabitants. The scarceness of Anopheles mosquitoes in urban areas of Blantyre has previously been reportedusing pyrethrum space spray catches (PSCs), investigators collected a combined total of more than 900 An. gambiae s.l. and $A n$. funestus from two rural sites and only $2 A n$. funestus mosquitoes from an urban site. ${ }^{20}$

This study also supports the findings of the 2010 Demographic and Health Survey (DHS), ${ }^{21}$ those reported generally from several major cities in sub-Saharan Africa, ${ }^{11,22}$ as well as the findings of Mathanga and others, ${ }^{9,23}$ who demonstrated that malaria cases in urban Blantyre were more likely to have a history of recent travel to rural areas.

It was not surprising to observe that anophelines were present in the study area at very low densities of $10.3 \%$ and $12.4 \%$ in 2008 and 2010, respectively. The majority of mosquitoes found inside study households were culicines, as these mosquitoes are adapted to urban environments characterised by organically polluted water habitats. Although molecular identifications by PCR were unsuccessful on a large proportion of the specimens ( $\mathrm{n}=34,70.8 \%$ ), the detection of An. funestus s.s., an important and efficient malaria vector in sub-Saharan Africa (including Malawi), was an important finding in the study, as it was primary evidence of the potential for malaria disease transmission in the study area. The excess PCR failures could have been attributable two factors. The primary reason may have been that DNA quality was compromised by DNA degradation, as specimens were

Table 3: Multivariate analysis of factors associated with Anopheles abundance using Poisson regression

\begin{tabular}{|c|c|c|c|c|}
\hline \multirow{2}{*}{ Variable } & \multicolumn{2}{|l|}{2008} & \multicolumn{2}{|l|}{2010} \\
\hline & IRR (95\% CI) & P-value & IRR $(95 \%$ CI $)$ & P-value \\
\hline ITNs (Yes) & $0.78(0.39$ to 1.53$)$ & 0.47 & $0.97(0.82$ to 1.15$)$ & 0.73 \\
\hline ITNs (No) & Reference & & Reference & \\
\hline Windows (Yes) & $0.78(0.39$ to 1.53$)$ & 0.099 & $1.1(0.64$ to 1.79$)$ & 0.80 \\
\hline Windows (No) & Reference & & Reference & \\
\hline Eaves (Yes) & $3.5(1.5$ to 8.1$)$ & 0.004 & $2.08(1.43$ to 3.04$)$ & $<0.001$ \\
\hline Eaves (no) & Reference & & Reference & \\
\hline Altitude & 0.997 (0.996 to 0.998$)$ & $<0.001$ & $1.002(1.001$ to 1.002$)$ & $<0.001$ \\
\hline
\end{tabular}

$\mathrm{IRR}=$ incidence rate ratio; $\mathrm{CI}=$ confidence interval; $\mathrm{ITN}=$ insecticide-treated net 
stored dry, using a desiccant (silica gel), and samples were not processed immediately. It is also possible that some of the specimens that did not amplify were sibling species whose primers were not included in the primer cocktail used in the PCR identifications. For instance, a study carried out in Karonga, northern Malawi, showed the presence of an $A n$. funestus-like mosquito, described as a new species within this group of mosquitoes. ${ }^{24}$

Several factors (presence of open house eaves, altitude, window type, and ITN ownership) were associated with the incidence of Anopheles mosquitoes. In univariate analyses, houses with open eaves and open windows were associated with an increased incidence of indoor resting Anopheles mosquitoes, corroborrating previous studies that have shown that houses with open eaves permit mosquito entry. ${ }^{25,26}$ We did not assess the presence of ceilings, but these have also been shown to reduce mosquito entry in houses in urban areas ${ }^{27}$ and could have contributed to the absence of anopheline mosquitoes in urban areas of Blantyre as well.

Ownership of ITNs was not associated with a reduced number of mosquitoes in either survey. The use of ITNs has repeatedly been shown to reduce the number of mosquitoes inside houses ${ }^{28-30}$, so the lack of a statistically significant impact was somewhat surprising. It is possible that net use was so high in both urban and rural areas that there was limited statistical power to detect a difference between households with and without bednets. Furthermore, we did not assess the condition and insecticide levels of the nets. Both surveys were conducted before long-lasting nets were widely available, and the nets may therefore have been inadequately treated. It is also possible that An. funestus populations sampled in the study area were resistant to the deltamethrin (a pyrethroid insecticide) used to treat ITNs, and that could partly explain the lack of association between ITN ownership and vector density in the study households. Unfortunately, this study did not assess the resistance status of vector populations in the study area.

Our study showed conflicting results when elevation was analysed in the two surveys. The number of An. funestus increased at lower elevations during the first survey, while in the second survey, numbers increased with increasing elevation. The finding of increased altitude being associated with increased incidence of An. funestus during the second survey was unexpected. Anopheles mosquitoes are generally more prevalent in lower altitudes, where standing water is more abundant. It is possible that there was more standing water at lower elevations during the wet season but more standing water at high elevations in the dry season as streams dry up and leave pools of water. However, because we did not assess the availability of potential larval habitats in this study, it is difficult to conclusively explain the observations and the ecological parameters that governed mosquito breeding and presence.

It was not surprising to find Culex spp. constituting the bulk of mosquitoes sampled in the study area. This mosquito species is adapted to urban environments, as it proliferates in breeding habitats rich in organic matter. However, the presence of Mansonia spp., though in very low numbers, is worth highlighting. Mansonia mosquitoes breed in swampy environments, where water plants are prevalent, and therefore their presence in the study area pointed to the existence of such localised habitats. Both Culex spp. and Mansonia spp. have been implicated in the transmission of lymphatic filariasis, caused by Wuchereria bancrofti, in Chikwawa District, southern Malawi. ${ }^{31}$

The absence of anopheline mosquitoes in urban areas of this study contrasts with observations from other urban settings, where transmission was present in urban areas, even if it was indeed lower than transmission in rural areas. In most of these studies, transmission was generally linked to clearly defined breeding sites and declined at increasing distances from these sites. In a study carried out in urban Dakar, vectors decreased with increasing distance from a large breeding site. ${ }^{32}$ In Accra, Ghana, ${ }^{8}$ breeding of $A n$. gambiae s.s. was found to be associated with urban agriculture, with an EIR of 19.2 infectious bites per person per year (ibppy) near agricultural areas, compared to 6.6 ibppy at sites further from agricultural areas. In Lagos, Nigeria, ${ }^{33}$ and in Dar es Salaam, Tanzania, ${ }^{34}$ An. gambiae s.l. larvae have been found breeding in polluted water bodies within urban environments, suggesting that mosquitoes have adapted to the changing conditions in these environments. These studies highlight the diversity of urban environments and the diversity of responses of Anopheles mosquitoes to the anthropogenic changes related to urbanisation.

Failure to detect indoor resting Anopheles mosquitoes from houses located in urban areas in the present study could be due to a number of factors. First, it is possible that there is no Anopheles breeding within urban areas of Blantyre inside city boundaries. The lack of breeding could result from unfavourable breeding habitats due to organic pollution, although this study did not assess the availability or water quality of potential larval habitats. Furthermore, many houses had closed windows and eaves, meaning that even if breeding habitats were available, human hosts may not have been available, or mosquitoes may have been resting outdoors because of the changes in house construction. Additionally, this study was carried out over a relatively short period of time. In an environment where Anopheles mosquitoes are rare, more effort may be required over time and space to detect the few and sparse mosquitoes present. Finally, all efforts to collect Anopheles mosquitoes in the study area relied on a single collection technique (the backpack aspirator) to collect indoor resting mosquitoes. Multiple collection techniques, targeting the various species with different resting behaviour characteristics, may be required to detect low densities of Anopheles mosquitoes, particularly if there are behavioural changes associated with differences in housing construction. Further studies targeting areas that may likely hold potential breeding sites may provide further insight into the potential for malaria transmission within urban Blantyre.

\section{Conclusions}

The detection of An. funestus s.s. and, to a lesser extent, An. gambiae s.l. within the periphery of Blantyre city was indicative of malaria transmission taking place in these rural areas. However, we were unable to detect either species within urban Blantyre. This study confirms previous findings suggesting that much of the malaria burden in Blantyre city results from movement of urban residents to and from rural areas. There is strong need to fully understand the ecological and environmental factors that support breeding of Anopheles mosquitoes found in the peripheral areas surrounding the city boundaries. 


\section{Acknowledgements}

Field work and data collection was ably carried out with assistance from the Blantyre District Health Office. We would like to acknowledge the following district environmental health officers who participated in the data collection exercise: Mr Sunganani Namelo, Mrs Sebastiana Sambo, Mr Lonnex Chalwita, Mr Dikirani Chadza, Mr Samson Kumphale, and Mr Alinafe Hauya. The authors would like to kindly acknowledge the numerous home owners and the communities in which they lived for granting us permission to enter their homes to collect mosquitoes.

This study was made possible with funding from the Cooperative Agreement Number 3U01CK000135 between the Centers for Disease Control and Prevention and the University of Malawi, and NIH Grant Number 5R01TW7599.

\section{Conflicts of interest}

The authors declare that they have no conflicts of interest related to this study.

\section{References}

1. Wang SJ, Lengeler C, Mtasiwa D, Mshana T, Manane L, Maro G, et al. Rapid Urban Malaria Appraisal (RUMA) II: epidemiology of urban malaria in Dar es Salaam (Tanzania). Malar J. 2006;5:28.

2. Wang SJ, Lengeler C, Smith TA, Vounatsou P, Akogbeto M, Tanner M. Rapid Urban Malaria Appraisal (RUMA) IV: epidemiology of urban malaria in Cotonou (Benin). Malar J. 2006;5:45.

3. Wang SJ, Lengeler C, Smith TA, Vounatsou P, Cisse G, Tanner M. Rapid Urban Malaria Appraisal (RUMA) III: epidemiology of urban malaria in the municipality of Yopougon (Abidjan). Malar J. 2006;5:29.

4. Wang SJ, Lengeler C, Smith TA, Vounatsou P, Diadie DA, Pritroipa X, et al. Rapid urban malaria appraisal (RUMA) I: epidemiology of urban malaria in Ouagadougou. Malar J. 2005;4:43.

5. Omumbo JA, Guerra CA, Hay SI, Snow RW. The influence of urbanisation on measures of Plasmodium falciparum infection prevalence in East Africa. Acta Trop. 2005 Jan;93(1):11-21.

6. Dongus S, Nyika D, Kannady K, Mtasiwa D, Mshinda H, Gosoniu L, et al. Urban agriculture and Anopheles habitats in Dar es Salaam, Tanzania. Geospat Health. 2009 May;3(2):189-210.

7. Klinkenberg E, McCall PJ, Hastings IM, Wilson MD, Amerasinghe FP, Donnelly MJ. Malaria and irrigated crops, Accra, Ghana. Emerg Infect Dis. 2005 Aug;11(8):1290-3.

8. Klinkenberg E, McCall P, Wilson MD, Amerasinghe FP, Donnelly MJ. Impact of urban agriculture on malaria vectors in Accra, Ghana. Malar J. 2008;7:151.

9. Mathanga DP, Campbell CH, Taylor TE, Barlow R, Wilson ML Reduction of childhood malaria by social marketing of insecticide-treated nets: a case-control study of effectiveness in Malawi. Am J Trop Med Hyg. 2005 Sep;73(3):622-5

10. Baragatti M, Fournet F, Henry MC, Assi S, Ouedraogo H, Rogier $\mathrm{C}$, et al. Social and environmental malaria risk factors in urban areas of Ouagadougou, Burkina Faso. Malar J. 2009;8:13.

11. Siri JG, Wilson ML, Murray S, Rosen DH, Vulule JM, Slutsker L, et al. Significance of travel to rural areas as a risk factor for malarial anemia in an urban setting. Am J Trop Med Hyg. 2010 Mar;82(3):391-7.

12. Kazembe LN, Appleton CC, Kleinschmidt I. Spatial analysis of the relationship between early childhood mortality and malaria endemicity in Malawi. Geospat Health. 2007 Nov;2(1):41-50.

13. Kazembe LN, Kleinschmidt I, Sharp BL. Patterns of malaria-related hospital admissions and mortality among Malawian children: an example of spatial modelling of hospital register data. Malar J. 2006;5:93.

14. Kantsitsi P. Upgrading trading centres surrounding Blantyre City A strategy for attaining sustainable urbanization. In. Blantyre, Malawi: Blantyre District Assembly; 2015.

15. Vanden Eng JL, Wolkon A, Frolov AS, Terlouw DJ, Eliades MJ, Morgah K, et al. Use of handheld computers with global positioning systems for probability sampling and data entry in household surveys. The American journal of tropical medicine and hygiene. 2007 Aug;77(2):393-9.
16. Gillies MT, Coetzee M. A supplement to the Anophelinae of Africa south of the Sahara (Afrotropical Region). Publications of the South African Institute for Medical Research No. 55. The South African Institute for Medical Research; 1987.

17. Koekemoer LL, Kamau L, Hunt RH, Coetzee M. A cocktail polymerase chain reaction assay to identify members of the Anopheles funestus (Diptera: Culicidae) group. The American journal of tropical medicine and hygiene. 2002 Jun;66(6):804-11.

18. Scott JA, Brogdon WG, Collins FH. Identification of single specimens of the Anopheles gambiae complex by the polymerase chain reaction. The American journal of tropical medicine and hygiene. 1993 Oct;49(4):520-9. 19. Wilkins EE, Howell PI, Benedict MQ. IMP PCR primers detect single nucleotide polymorphisms for Anopheles gambiae species identification, Mopti and Savanna rDNA types, and resistance to dieldrin in Anopheles arabiensis. Malaria journal. 2006;5:125.

20. Sexton JD, Tambala P. Activity Report No. 58: Entomological assessment in Blantyre District, Malawi, in support of an Insecticide-Treated Materials Project, Division EH. Contract No. HRN-5994-C-00-3036-00, Project No. 936-5994; 1999

21. National Statistical Office \& ICF Macro. Malawi demographic and health survey 2010. Zomba, Malawi, and Calverton, Maryland, USA: NSO and ICF Macro; 2011.

22. Pond BS. Malaria indicator surveys demonstrate a markedly lower prevalence of malaria in large cities of sub-Saharan Africa. Malar J. 2013 Sep 10;12:313

23. Mathanga DP, Campbell CH, Taylor TE, Barlow R, Wilson ML. Socially marketed insecticide-treated nets effectively reduce Plasmodium infection and anaemia among children in urban Malawi. Trop Med Int Health. 2006 Sep;11(9):1367-74.

24. Spillings BL, Brooke BD, Koekemoer LL, Chiphwanya J, Coetzee M, Hunt RH. A new species concealed by Anopheles funestus Giles, a major malaria vector in Africa. The American journal of tropical medicine and hygiene. 2009 Sep;81(3):510-5.

25. Lindsay SW, Snow RW. The trouble with eaves; house entry by vectors of malaria. Trans R Soc Trop Med Hyg. 1988;82(4):645-6.

26. Lindsay SW, Emerson PM, Charlwood JD. Reducing malaria by mosquito-proofing houses. Trends Parasitol. 2002 Nov;18(11):510-4.

27. Ogoma SB, Lweitoijera DW, Ngonyani H, Furer B, Russell TL, Mukabana WR, et al. Screening mosquito house entry points as a potential method for integrated control of endophagic filariasis, arbovirus and malaria vectors. PLoS Negl Trop Dis. 2010;4(8):e773.

28. Gimnig JE, Kolczak MS, Hightower AW, Vulule JM, Schoute E, Kamau $\mathrm{L}$, et al. Effect of permethrin-treated bed nets on the spatial distribution of malaria vectors in western Kenya. Am J Trop Med Hyg. 2003 Apr;68(4 Suppl):115-20.

29. Lindblade KA, Gimnig JE, Kamau L, Hawley WA, Odhiambo F, Olang $\mathrm{G}$, et al. Impact of sustained use of insecticide-treated bednets on malaria vector species distribution and culicine mosquitoes. J Med Entomol. 2006 Mar;43(2):428-32.

30. Mbogo CN, Baya NM, Ofulla AV, Githure JI, Snow RW. The impact of permethrin-impregnated bednets on malaria vectors of the Kenyan coast. Med Vet Entomol. 1996 Jul;10(3):251-9.

31. Merelo-Lobo AR, McCall PJ, Perez MA, Spiers AA, Mzilahowa T, Ngwira B, et al. Identification of the vectors of lymphatic filariasis in the Lower Shire Valley, southern Malawi. Trans R Soc Trop Med Hyg. 2003 May-Jun;97(3):299-301.

32. Trape JF, Lefebvre-Zante E, Legros F, Ndiaye G, Bouganali H, Druilhe $\mathrm{P}$, et al. Vector density gradients and the epidemiology of urban malaria in Dakar, Senegal. The American journal of tropical medicine and hygiene. 1992 Aug;47(2):181-9.

33. Awolola TS, Oduola AO, Obansa JB, Chukwurar NJ, Unyimadu JP. Anopheles gambiae s.s. breeding in polluted water bodies in urban Lagos, southwestern Nigeria. J Vector Borne Dis. 2007 Dec;44(4):241-4.

34. Sattler MA, Mtasiwa D, Kiama M, Premji Z, Tanner M, Killeen GF, et al. Habitat characterization and spatial distribution of Anopheles sp. mosquito larvae in Dar es Salaam (Tanzania) during an extended dry period. Malar J. 2005 Jan 14;4:4. 